

\title{
Internet como herramienta transformadora de las relaciones familiares entre adultos mayores y sus familiares migrantes ${ }^{1}$ Internet as a tool for transforming family relationships between older adults and family migrants
}

\author{
Julián Enrique Páez Valdez², Claudia Lorena Hernández Hincapiéz \\ Universidad del Quindío, Armenia - Quindío, Colombia \\ Universidad Católica de Pereira, Pereira, Colombia
}

Artículo recibido en marzo de 2016; artículo aceptado en junio de 2016

Citación del artículo: PAÉZ J. \& HERNÁNDEZ C. (2016). Internet como herramienta transformadora de las relaciones

familiares entre adultos mayores y familiares migrantes. I+D Revista de Investigaciones, 8 (2), pp. 6 - 14

\begin{abstract}
Resumen
En una sociedad globalizada y cada vez más dirigida hacia los avances tecnológicos, los nuevos instrumentos, capaces de modificar nuestra forma de comunicarnos, ha transformado diversos aspectos sociales. En este sentido, esta investigación, de tipo cualitativo, estudia los usos y las apropiaciones de la internet por parte de los adultos mayores del municipio de Montenegro, Quindío (Colombia), entendida esta como una herramienta capaz de mantener y transformar las relaciones familiares con quienes se encuentran en el extranjero. La investigación permite analizar los nuevos significados de la internet en el ámbito familiar, específicamente para el adulto mayor, ya que cuando se presenta el fenómeno migratorio la satisfacción de las necesidades comunicativas es trascendental. También se describe el cambio de los roles familiares debidos al fenómeno migratorio, se identifican los rituales que se asumen en cuanto a la utilización del computador y por consiguiente de la internet, y el sentido que le da el adulto mayor a la comunicación con su familiar migrante. La metodología utilizada es cualitativa, de enfoque histórico hermenéutico y basada en el uso de entrevistas estructuradas abiertas y observaciones participantes, que permiten a los investigadores adentrarse en la realidad de los sujetos de investigación.
\end{abstract}

Palabras clave: migración, internet, vejez, uso y apropiación de medios, relaciones familiares.

\begin{abstract}
In a globalized society and increasingly directed to technological advances, creating new instruments capable of transforming the way we communicate, has managed to involve or restrict various aspects and characters, it is for this reason that this qualitative study, study uses and appropriations that have the elderly of the municipality of Montenegro, Colombia, regarding the Internet as a tool capable of maintaining and transform family relationships between them and their relatives who are abroad. This research allows us to analyze the new 1. El presente artículo es resultado del trabajo de investigación cualitativo denominado: Usos y apropiaciones de la internet, como herramienta transformadora de las relaciones familiares entre el adulto mayor y su familiar migrante en el municipio de Montenegro, Quindío, proyecto perteneciente al grupo de Investigación en Comunicación, Cultura y Periodismo del programa de Comunicación Social - Periodismo de la Universidad del Quindío, de la ciudad de Armenia (Colombia). Dirección: Carrera 15, Calle 12 Norte, Armenia - Quindío, +6 7359300

2. Comunicador social - periodista, Universidad del Quindío. Magíster en Ciencias Humanas, mención Información y Comunicación, Universidad Sorbonne Nouvelle - París III. Docente- investigador del grupo Comunicación, Cultura y Periodismo de la Universidad del Quindío de la ciudad de Armenia (Colombia) y docente- investigador, líder del grupo de investigación Comunicación, Educación y Cultura de la Universidad Católica de Pereira. (Colombia) jepaez@uniquindio.edu.co julian.paez@ucp.edu.co

3. Comunicadora social - periodista, Universidad del Quindío. Licenciada en lenguas modernas (en formación), Universidad del Quindío. Comunicadora organizacional y productora audiovisual.Claudialorena_1788@hotmail.com
\end{abstract}


meanings given to the Internet at the family level, specifically for the Elderly, since when the migration occurs communicative needs are transcendental. This article also describes the changing family roles by migration, identifies the rituals that are assumed regarding the use of the device (computer) and therefore of the Internet and the sense that it gives the elderly to communicate with their migrant family. The methodology used is qualitative, hermeneutical historical approach and based on the use of structured interviews open and participant observations, which allow researchers to delve into the reality of research subjects, allowing the participation.

Keywords: Migration, Internet, Old Age, Use and Appropiation Media, Familiar Relationship.

El proceso migratorio implica la separación familiar y social de una persona que decide irse de su residencia habitual para mejorar la calidad de vida de los integrantes de su hogar, o por otros motivos. Este fenómeno ha generado nuevas dinámicas familiares y nuevas necesidades comunicativas, que, de alguna manera, vienen a ser solucionadas por la tecnología, como el celular o la internet, instrumentos que dejan de ser meras herramientas para convertirse en medios de comunicación gracias al uso y la apropiación que de ellos hacen las personas para satisfacer sus necesidades, en este caso, comunicativas, afectivas y de sostenimiento de las relaciones familiares.

La tendencia mundial de migración va en aumento; en Colombia, la cifra de personas fuera del país es de 4700 000, según la Cancillería (2012); la mayoría de migrantes colombianos se dirigen principalmente hacia España, Estados Unidos y Venezuela. Este fenómeno ha cambiado la organización de la familia en cuanto a los roles que desempeñan cada uno de sus integrantes. Un ejemplo de este cambio es el del adulto mayor, que pasó de ser pasivo a activo; a esto se suman unas nuevas formas de organización familiar, llamadas por Dulcey (2002) "famimultigeneracionales", es decir, hogares constituidos indistintamente, pero que conservan un imaginario de familia, como por ejemplo, madres solteras, padrastros, madrastras, hijos adoptivos, hijos de hogares divorciados, parejas homosexuales, padres sustitutos como los abuelos, entre otros.

La familia sigue siendo para Colombia la base del desarrollo psíquico, físico, psicológico y social de las personas, sin importar cómo esté constituida; por lo tanto, la necesidad de sostenimiento de las relaciones familiares lleva a los adultos mayores a buscar alternativas, tanto internas como externas, para mantener la organización familiar que se había constituido antes de que se fuera el familiar migrante, y que debe mantenerse ahora que está ausente.

En este estudio es especialmente importante el adulto mayor, ya que es él quien queda a cargo del hogar que el migrante abandonó y pasa a ser un adulto mayor activo, con responsabilidades de crianza de los nietos y administración del dinero enviado por el migrante; además se constituye en el garante del sostenimiento del hogar - como sea que esté constituido-. El adulto mayor es, en consecuencia, quien tiene mayores necesidades comunicativas, aunque esto depende del grado de cercanía afectiva y de consanguinidad que tenga con quien está en la distancia, como ocurre con hijos, nietos, hermanos y esposos, que suelen ser los más importantes para él.

En este sentido, es importante observar de qué manera los adultos mayores afrontan el fenómeno migratorio como un proceso que puede modificar algunas conductas comunicativas y por consiguiente, el proceso mismo de la constitución familiar. En consecuencia, abordar el fenómeno migratorio permite comprender a qué responde y cómo influye en las familias.

\section{El adulto mayor en ambientes migratorios}

Al hablar de fenómenos migratorios es muy recurrente asociar dicho término al hecho de abandonar el país de origen para asentarseenotraentidad administrativamente diferente; sin embargo, es importante reconocer que existen distintos acercamientos a este fenómeno y que podríamos resumir en tres definiciones fundamentales: migración, emigración e inmigración.

Denominamos entonces migración a todo el desplazamiento poblacional que se produce desde un lugar de origen a un lugar de destino y que lleva consigo un cambio de la residencia habitual; en términos de la OIM -Organización Internacional para las Migraciones-: "Movimiento de población hacia el territorio de otro Estado o dentro del mismo que abarca todo movimiento de personas sea cual fuere su tamaño, su composición o sus causas; incluye migración de refugiados, personas desplazadas, personas desarraigadas, migrantes económicos" (OIM, 2006, p.38).

Por otra parte, existe el concepto de emigración, el cual es reconocido por la OIM (2006) como cualquier acto que incluya la salida de un territorio con el fin de asentarse en otro, teniendo en cuenta que cualquier ciudadano tiene el derecho de salir de su país, a no ser que incurra 
en delitos o faltas que estén gravadas en los sistemas judiciales de cada país.

Mientras que el concepto de inmigración, entendido en definiciones de la OIM (2006) es el ingreso de cualquier ciudadano a un Estado del cual no es ciudadano.

Estas definiciones guardan estrecha relación con dos conceptos muy relevantes para los estudios de este tipo: el de territorio y el de frontera, puesto que en muchas ocasiones hemos ligado los conceptos migratorios exclusivamente a las delimitaciones espaciales hechas por los Estados, dejando por fuera aspectos como los desplazamientos internos y los mismos procesos migratorios que viven las familias en el interior de sus hogares, al igual que desplazamientos más cercanos que podrían considerarse migratorios, ya que se rompe la frontera de la cercanía y se empiezan a construir nuevos territorios. Es así como el hijo que abandona su casa por primera vez crea un universo territorial nuevo y por consiguiente, establece fronteras con los que inicialmente formaban su núcleo discursivo y social.

\section{La internet como herramienta mediadora para las familias}

Al ser un medio que contiene los demás medios existentes, la internet genera un cambio en la concepción del tiempo y del espacio para el que tiene o no esta herramienta, lo que McLuhan (1996) Ilama brecha digital de las relaciones entre las personas y la globalización. En el caso de los hogares con experiencia migratoria, la internet se convierte en un elemento que garantiza la comunicación hablada, visual y escrita con el migrante, en una forma de materialización de un deseo perpetuo de saber cómo está el familiar. No obstante, para los adultos mayores una de las desventajas de la internet es que genera una ilusión de presencia del familiar, aun cuando este se encuentre a miles de kilómetros de su lugar de origen.

La internet viene a ser un puente conector para la solidificación afectiva y simbólica de las relaciones familiares y el garante de que el migrante, así se encuentre físicamente fuera del hogar, pueda intervenir en decisiones que se tomen con el dinero enviado (remesa) y con los problemas que se tengan en la casa de origen. Esta herramienta tecnológica que, mediada por un grupo se convierte en medio de comunicación, permite la interacción y el acortamiento de las distancias y el tiempo entre el familiar que se fue y los que se quedan.

Los hogares montenegrinos con experiencia migratoria que tienen internet en su casa son pocos; sin embargo, las familias que no cuentan con este servicio en sus hogares también se han servido alguna vez de la internet, bien sea en salas públicas o en casas de familiares cercanos. Los adultos mayores han usado la internet para ocasiones especiales como un cumpleaños, o para dar a conocer un nuevo integrante de la familia, lo que la convierte, así sea en menor medida, en una herramienta tecnológica reconocida y de importancia para el grupo familiar en encuentros ocasionales.

\section{Formulación del problema}

Al unir cuatro elementos fundamentales, que son migración, adulto mayor, internet y relaciones familiares, se desemboca en un estudio profundo de la forma como la internet, de acuerdo al contexto en donde se utiliza, puede cambiar la familia, las relaciones, los roles y los poderes entre los integrantes del hogar, convirtiéndose en un medio cotidiano y ritual que genera nuevas formas de interacción en el hogar. Dicho estudio permite también analizar el uso y la apropiación que le puede dar el adulto mayor a una herramienta desconocida para él. La apropiación es entendida desde Crovi (2007) como el proceso de interiorización del uso de la herramienta, uso que está dado desde Jull (1982) como la parte introductoria para que las personas se apropien de un medio, es decir, la habilidad técnica para utilizar un aparato, la periodicidad y las experiencias que se tienen con la utilización de la herramienta como tal.

Esta es la razón por la que se hace más interesante el estudio de este fenómeno en el adulto mayor, quien es considerado en los estudios sobre brechas digitales como una persona inhabilitada y excluida para usar la herramienta, lo cual no quiere decir que no se pueda apropiar de ella y que no se le convierta en una necesidad en cuanto a cuestiones de comunicación con su familiar migrante. Según el Departamento Administrativo Nacional de Estadística (DANE, 2005), la ausencia de un familiar que se encuentra en el exterior genera una necesidad de comunicación por parte del familiar migrante de un $85 \%$, que en la mayoría de veces es suplida por el celular. Pero cuando se quiere cubrir una insuficiencia de comunicación más cercana, llega la internet como nueva herramienta que permite reducir fronteras, tanto espaciales como temporales, y aunque no alcanza hasta la cercanía física sí ofrece la comunicación audiovisual que el celular no permite. Del panorama expuesto surge una interrogante que es resuelta en esta investigación: ¿cómo el uso y la apropiación de la internet como herramienta de la comunicación y la información por parte del adulto mayor ha transformado las relaciones familiares con su familiar migrante? 


\section{Método}

\section{Tipo de estudio}

Para esta investigación se utilizó una metodología cualitativa y un tipo de investigación IAP (Investigación Acción Participación) con el propósito de trabajar a escala micro-social y, de manera más profunda, en los instrumentos aplicados a fin de responder a la pregunta formulada anteriormente. Lo primero que se hizo fue determinar las características de la población con la que se trabajaría, que para este estudio eran hombres o mujeres mayores de sesenta y cinco años, con familiares migrantes y que hubieran utilizado alguna vez en su vida la internet para comunicarse con su familiar migrante.

\section{Participantes}

Este proyecto tuvo una unidad de análisis conformada por diez adultos mayores con los cuales se utilizaron dos formatos de entrevista estructurada (Tabla 1 y Tabla 2) y un formato de observación participante (Tabla 3). Asimismo, se trabajaron guías exploratorias capaces de dar información a profundidad sobre las condiciones demográficas de los integrantes de la unidad de análisis y sus familiares migrantes. Además del encuentro con los diez adultos mayores, también se tuvieron en cuenta diez establecimientos en donde se presta el servicio de internet (salas de internet) y un instituto de educación no formal que se dedica a enseñar a los montenegrinos el manejo de la internet; con estos último se utilizó la entrevista estructurada - abierta.

Como este estudio es de tipo IAP, se realizaron visitas adicionales a los adultos mayores con el fin de hablar con ellos, compartir sus experiencias y que los investigadores se adentraran en el problema como tal. También se hizo registro fotográfico, de audio y se presenciaron algunos momentos de comunicación entre los adultos mayores y sus familiares, para tener una experiencia más cercana.

\section{Procedimiento - interpretación de la información}

\section{La internet. De lo práctico a lo íntimo}

Hablar de internet es hacer alusión a una idea tecnológica que proviene de un concepto sociológico y es el de las redes, ya que este instrumento, que en un principio se conoció como Arpanet y fue creado como una herramienta para apoyar la comunicación reticular de las unidades militares de Estados Unidos, evidenció su fortaleza como herramienta comunicativa y se abrió al público universitario, inicialmente. Es en esta idea sociológica de la construcción de redes en donde se encuentra la importancia teórica de hablar de la internet y su relación con los adultos mayores, ya que, a pesar de ser considerada una mera herramienta tecnológica, permite avances importantes en ese aspecto social que es la relación familiar, y el adulto mayor empieza a evidenciar en ella una utilidad no solo procedimental sino también una utilidad familiar, como herramienta para preservar los temas de conversación de los grupos familiares.

A través de su dinámica, el Internet propone un proceso de construcción y reconstrucción de "redes sociales", entendiendo éstas como sistemas abiertos mediante los cuales se produce intercambio dinámico entre sus propios integrantes como con integrantes de otros grupos y organizaciones, posibilitando así la potencialización de los recursos que poseen (Dabas,1995, p. 142).

El municipio de Montenegro, departamento de Quindío es una de las regiones con mayor nivel de migración de Colombia: según el DANE (2005) 6,8\% de los adultos mayores en cuyas familias se presenta el fenómeno migratorio cumplen unas características que otras personas de estas edades no tienen, a saber: una relación directa o indirecta con la internet, directa en el sentido de que la tienen en la casa e indirecta cuando la herramienta se encuentra en las salas de internet o en las casas de otros familiares cuyos parientes también son migrantes. Esto quiere decir que la mayoría de las personas mayores de sesenta y cinco años de este municipio cuentan con el acceso a la herramienta (pública o privada) y, por lo tanto, conocen de su existencia; sin embargo, ¿será que los adultos mayores la usan y se apropian de ella para comunicarse con su familiar migrante?

El concepto de uso, según Jull (1982), hace referencia a los primeros acercamientos que tiene una persona hacia una herramienta, su utilización técnica y las habilidades que tiene, la periodicidad (uso estructural) y quién la maneja (uso relacional).

Se observa que en los hogares con experiencia migratoria los nietos son quienes usan la internet y facilitan la comunicación al adulto mayor con el migrante, razón por la cual el adulto mayor no ha querido aprender a manipular la herramienta y no se apropia de ella, es decir, no la hace parte de su vida cotidiana, así para él la comunicación sea indispensable. Este hecho hace que para el adulto mayor el celular sea el medio más fácil y efectivo para interactuar con su familiar migrante, por ser quien tiene el poder sobre el aparato (celular) y siempre lo tiene a su servicio. Este proceso interpretativo se derivó del análisis de la primera entrevista realizada, que se puede ver en la Tabla 1. 
Los adultos mayores son personas que tienen edades superiores a los 65 años y al no haber nacido con la tecnología, desconocen la internet más que cualquier otro grupo generacional. Este grupo poblacional configura lo

\section{Tabla 1}

Formato primera entrevista estructurada

Análisis de la primera entrevista
Pregunta
Nombre del entrevistado, edad

que McLuhan (1996) define como emigrantes digitales, es decir personas a las que se les hace más difícil el uso y la apropiación de esta herramienta. En el caso de este estudio, se pudo observar que a pesar de que en la mayoría de los hogares con experiencia migratoria se cuenta con este servicio (público o privado), los conocimientos que los adultos mayores tienen sobre la herramienta fueron adquiridos por iniciativa externa, es decir por recomendación o sugerencia de alguien, que en su mayoría de veces es el familiar migrante.

\section{El adulto mayor: de persona pasiva a activa en la familia}

Tener sesenta y cinco años de edad, según la Organización de Naciones Unidas (ONU, 2005), representa unos cambios para la persona, ya que este es el punto culminante del proceso de envejecimiento natural que presentan los seres humanos; es una esta etapa en la que se presentan cambios tanto en lo intelectual, psicológico, cultural como en la parte biológica y física. Puede el adulto mayor estar lúcido y saludable o también puede tener una vejez llena de quebrantos de salud y con problemas sicológicos, entre otros; todo depende del cuidado que haya tenido en el proceso de envejecimiento cronológico (Sánchez, 2009). Sin embargo, la esperanza de vida en Colombia se ha incrementado y se hace todo lo posible por prolongarla y mejorarla. Además, para que la calidad de vida del adulto mayor sea óptima, es indispensable la familia.

Al pasar de una vida externa (amigos, trabajo) a una vida interna (familiar), la familia se convierte en parte esencial de la vida del adulto mayor y de la de cada uno de los individuos que componen el núcleo familiar (Páez, 1984), ya que en él debe encontrar la satisfacción de las necesidades físicas: alimentación y vivienda; psíquica: amor, autoestima y equilibrio mental; y sociales: pertenencia a algún grupo (familia), comunicación e identificación.

En los hogares actuales donde el adulto mayor está presente, se han experimentado unos cambios trascendentales en lo que tiene que ver con las responsabilidades del hogar y los roles, pues este pasó de ser una persona asistida por sus hijos a asistir a los hijos de sus hijos. Todo este proceso de transformación de las familias tiene su por qué: los cambios económicos, tecnológicos, migratorios e industriales que han hecho que las familias se potencialicen. Este proceso interpretativo se derivó del análisis de la segunda entrevista realizada, que se puede ver en la Tabla 2.

En este estudio es importante el fenómeno migratorio pues es un factor que ha incidido en la reestructuración de las familias del municipio de Montenegro, es decir, el emigrante pasa a ser la persona emisora de dinero mientras el adulto mayor pasa a ser el receptor y administrador de dichos recursos, al tiempo que se convierte en el padre sustituto de sus nietos, ya que en la mayoría de los casos observados en esta investigación es el hijo del adulto mayor el que se va para mejorar la calidad de vida de la familia.

Es de resaltar que la adulta mayor (madre del hijo migrante) es la que pasa a ser la cabeza de la familia en lo que tiene que ver con la administración del dinero: es ella quien decide qué se compra y qué no, qué mejoras se le hacen a la casa y por último, es quien toma decisiones sobre la crianza de los nietos, muchas de estas en conjunto con el familiar migrante. Por lo tanto, las mujeres son las que tienen sobrecarga de responsabilidades en la casa, porque pasan a ser las voceras, mientras que el adulto mayor hombre pasa a cumplir labores secundarias como arreglar daños en las viviendas, aunque también tiene a su cargo la crianza de los nietos ya que ha dejado de 
Tabla 2

\section{Formato segunda entrevista estructurada}

\begin{tabular}{|c|c|c|c|}
\hline \multicolumn{4}{|c|}{$\begin{array}{l}\text { Análisis de la segunda entrevista } \\
\text { Nombre del entrevistado, edad }\end{array}$} \\
\hline Pregunta & Respuesta & Interpretación & $\begin{array}{l}\text { Análisis por } \\
\text { categorías }\end{array}$ \\
\hline $\begin{array}{l}\text { 1. ¿Cuánto hace } \\
\text { que conoce la } \\
\text { internet y por qué } \\
\text { comenzó a usarla? }\end{array}$ & \begin{tabular}{lr}
\multicolumn{2}{l}{ Sistematización } \\
directa de la \\
respuesta del \\
entrevistado.
\end{tabular} & $\begin{array}{l}\text { Interpretación de la } \\
\text { respuesta según los } \\
\text { investigadores. }\end{array}$ & $\begin{array}{l}\text { La categoría de } \\
\text { esta pregunta es la } \\
\text { internet como medio } \\
\text { de communicación. }\end{array}$ \\
\hline $\begin{array}{l}\text { 2. ¿Aparte de } \\
\text { comunicarse con } \\
\text { su familiar, para } \\
\text { qué otra cosa } \\
\text { utiliza la internet? }\end{array}$ & \begin{tabular}{l}
\multicolumn{2}{l}{ Sistematización } \\
directa de la \\
respuesta del \\
entrevistado.
\end{tabular} & $\begin{array}{l}\text { Interpretación de la } \\
\text { respuesta según los } \\
\text { investigadores. }\end{array}$ & $\begin{array}{l}\text { La categoría de } \\
\text { esta pregunta es } \\
\text { uso y apropiación de } \\
\text { la internet. }\end{array}$ \\
\hline $\begin{array}{l}\text { 3. ¿Sería capaz de } \\
\text { vivir sin internet? }\end{array}$ & \begin{tabular}{lr}
\multicolumn{2}{l}{ Sistematización } \\
directa de la \\
respuesta del \\
entrevistado.
\end{tabular} & $\begin{array}{l}\text { Interpretación de la } \\
\text { respuesta según los } \\
\text { investigadores. }\end{array}$ & $\begin{array}{l}\text { La categoría de } \\
\text { esta pregunta es } \\
\text { uso y apropiación de } \\
\text { la internet, pero se } \\
\text { enfoca en la } \\
\text { apropiación. }\end{array}$ \\
\hline $\begin{array}{l}4 . \\
\text { cambiarían sCómo } \\
\text { r e I a c i o n e s } \\
\text { familiares si su } \\
\text { familiar regresara } \\
\text { del todo a } \\
\text { Colombia? }\end{array}$ & \begin{tabular}{lr}
\multicolumn{2}{l}{ Sistematización } \\
directa de la \\
respuesta del \\
entrevistado.
\end{tabular} & $\begin{array}{l}\text { Interpretación de la } \\
\text { respuesta según los } \\
\text { investigadores. }\end{array}$ & $\begin{array}{l}\text { La categoría de } \\
\text { esta pregunta es la } \\
\text { comunicación en la } \\
\text { edad adulta. }\end{array}$ \\
\hline $\begin{array}{l}\text { 5. ¿Le gustaría } \\
\text { aprender a usar la } \\
\text { internet para } \\
\text { comunicarse } \\
\text { pedir ayuda? }\end{array}$ & $\begin{array}{l}\text { Sistematización } \\
\text { directa de la } \\
\text { respuesta del } \\
\text { entrevistado. }\end{array}$ & $\begin{array}{l}\text { Interpretación de la } \\
\text { respuesta según los } \\
\text { investigadores. }\end{array}$ & $\begin{array}{l}\text { La categoría de } \\
\text { esta pregunta es } \\
\text { uso y apropiación de } \\
\text { la internet. }\end{array}$ \\
\hline $\begin{array}{l}\text { 6. ¿Usted considera } \\
\text { que el uso de la } \\
\text { internet ha } \\
\text { transformado las } \\
\text { relaciones de su } \\
\text { familia? }\end{array}$ & \begin{tabular}{lr}
\multicolumn{2}{l}{ Sistematización } \\
directa de la \\
respuesta del \\
entrevistado.
\end{tabular} & $\begin{array}{l}\text { Interpretación de la } \\
\text { respuesta según los } \\
\text { investigadores. }\end{array}$ & Objetivo general. \\
\hline
\end{tabular}

trabajar y no tiene pensión laboral para responder por el hogar.

Como parte del proceso investigativo se realizaron observaciones directas que permitieron responder a categorías y temas investigativos que no fueron evidentes durante las entrevistas; de esta manera se analizaron temas como los integrantes de la familia, las condiciones físicas del adulto mayor, los efectos de la migración en la transformación física del hogar, los ritos y mitos surgidos del uso de la internet, entre otros, que permitieron evidenciar el sentido que le dan los adultos mayores a la comunicación. Este proceso de análisis e interpretación se puede evidenciar en la siguiente ficha de observación directa, dividida en categorías: subtemas, observación y análisis (Tabla 3).

De acuerdo con lo interpretado a partir de la observación directa, podría decirse que las familias estudiadas se convierten en lo que Dulcey (2002) llama (citando a Dychtwaltd, 1995), familias multigeneracionales,
Tabla 3

Formato observación directa

\begin{tabular}{|c|c|c|c|}
\hline \multicolumn{4}{|c|}{$\begin{array}{c}\text { Análisis de la observación directa } \\
\text { Datos de la familia observada, edad del adulto mayor }\end{array}$} \\
\hline Categoría & Subtemas & Observación & Análisis \\
\hline \multirow{3}{*}{$\begin{array}{l}\text { La comunicación en } \\
\text { la edad adulta }\end{array}$} & $\begin{array}{l}\text { Integrantes de la } \\
\text { familia. }\end{array}$ & $\begin{array}{l}\text { Observación } \\
\text { realizada por los } \\
\text { investigadores. }\end{array}$ & $\begin{array}{c}\text { Análisis realizado por } \\
\text { los investigadores con } \\
\text { base en las categorías } \\
\text { propuestas. }\end{array}$ \\
\hline & $\begin{array}{l}\text { Condiciones físicas } \\
\text { del adulto mayor. }\end{array}$ & $\begin{array}{l}\text { Observación } \\
\text { realizada por los } \\
\text { investigadores. }\end{array}$ & $\begin{array}{c}\text { Análisis realizado por } \\
\text { los investigadores con } \\
\text { base en las categorías } \\
\text { propuestas. }\end{array}$ \\
\hline & $\begin{array}{l}\text { Efectos de la } \\
\text { migración en la } \\
\text { transformación } \\
\text { física del hogar. }\end{array}$ & $\begin{array}{l}\text { Observación } \\
\text { realizada por los } \\
\text { investigadores. }\end{array}$ & $\begin{array}{c}\text { Análisis realizado por } \\
\text { los investigadores con } \\
\text { base en las categorías } \\
\text { propuestas. }\end{array}$ \\
\hline \multirow{4}{*}{$\begin{array}{l}\text { El sentido de la } \\
\text { comucicación }\end{array}$} & $\begin{array}{l}\text { Autoridad y poder } \\
\text { familiar. }\end{array}$ & $\begin{array}{l}\text { Observación } \\
\text { realizada por los } \\
\text { investigadores. }\end{array}$ & $\begin{array}{c}\text { Análisis realizado por } \\
\text { los investigadores con } \\
\text { base en las categorías } \\
\text { propuestas. }\end{array}$ \\
\hline & $\begin{array}{l}\text { Disposición del } \\
\text { computador } \\
\text { dentro del hogar. }\end{array}$ & $\begin{array}{l}\text { Observación } \\
\text { realizada por los } \\
\text { investigadores. }\end{array}$ & $\begin{array}{c}\text { Análisis realizado por } \\
\text { los investigadores con } \\
\text { base en las categorías } \\
\text { propuestas. }\end{array}$ \\
\hline & $\begin{array}{l}\text { Ritos empleados } \\
\text { durante la } \\
\text { comunicación. }\end{array}$ & $\begin{array}{l}\text { Observación } \\
\text { realizada por los } \\
\text { investigadores. }\end{array}$ & $\begin{array}{c}\text { Análisis realizado por } \\
\text { los investigadores con } \\
\text { base en las categorías } \\
\text { propuestas. }\end{array}$ \\
\hline & $\begin{array}{l}\text { Mitos percibidos } \\
\text { durante la } \\
\text { conversación. }\end{array}$ & $\begin{array}{l}\text { Observación } \\
\text { realizada por los } \\
\text { investigadores. }\end{array}$ & $\begin{array}{c}\text { Análisis realizado por } \\
\text { los investigadores con } \\
\text { base en las categorías } \\
\text { propuestas. }\end{array}$ \\
\hline
\end{tabular}

conformadas por personas de varias familias, como ocurre en este caso: constituidas por abuelos y nietos o con una persona que no comparte consanguinidad, como nueras y yernos entre otros; sin embargo, la familia sigue siendo -como sea que esté constituida- la base de cada una de las personas que la conforman.

Al convertirse en la cabeza del hogar, el adulto mayor pasó de ser la persona pasiva de la familia a ser activa, ya que una de las necesidades actuales es que todas las personas de la familia cumplan actividades que la sostengan (Álvarez, 2000), y lo que interesa es el sostenimiento económico del hogar. Este hecho se evidencia, precisamente, en los hogares con práctica migratoria, que ven en las remesas un estado óptimo de la calidad de vida, pues entre más objetos se tengan mejor se vive. La posibilidad de que el migrante vuelva a su casa es remota, porque con su retorno se desequilibraría la estabilidad de la familia, no solo en el ámbito económico sino emocional; por ello, los migrantes solo vienen por temporadas, a pasar vacaciones, y se regresan, pues para su familia no es posible mantener el nivel de vida que han adquirido con las remesas si estas llegasen a faltar. 


\section{Triangulación de los resultados y análisis}

De acuerdo con Luhmann (1984), la sociedad requiere constantemente comunicarse y más aún cuando se es adulto mayor, etapa en que las necesidades de reconocimiento dentro de la familia se incrementan. Para el adulto mayor la internet no se convierte en una extensión de sus sentidos (McLuhan, 1996), si no que le atribuye ventajas frente a otros medios, ya que pueden ver a su familiar migrante y este lo puede ver a él, pero no se transforma en la extensión de sus necesidades de interacción, porque no es él quien se apropió de la internet ni tiene el poder frente a esta.

Por esta razón aparecen en el adulto mayor una serie de mitos relacionados con el uso de la internet, como su incapacidad para manejar una herramienta tecnológica que no creció con él e incapacidades intelectuales por no haber estudiado, que de una u otra forma predisponen a los adultos mayores a usar el objeto, razón por la cual no lo hacen parte de su vida cotidiana, así se tenga en la casa.

Específicamente, para hablar de los lugares de la casa y del computador, es necesario referirnos a dos casos en particular que son: los hogares de los adultos mayores que tienen computador, donde este ocupa un espacio jerárquico de carácter privado (Finol, 2006), porque son los hijos de los migrantes los que lo manejan, y está en sus cuartos; sin embargo, en algunas casas el computador se encuentra en el cuarto del migrante, que parece ser de uso privado en un primer momento, si bien el grupo familiar invita a sus familiares cercanos a que se conecten con sus familiares migrantes y pasa a convertirse en un equipo de uso público. Lo mismo sucede con los adultos mayores que no tienen internet: en el lugar al que acuden (familiares cercanos), la internet se convierte en un instrumento de uso público, independientemente de que tenga un carácter privado en el lugar que ocupa en la casa (cuartos y estudio).

En los hogares donde la comunicación por internet con el migrante es ocasional, el rito se visibiliza porque cada vez que hay una fecha especial, como un cumpleaños o el Día de la Madre entre otros, la familia entera se reúne a compartir ese momento con el familiar migrante, ya que la internet es el puente que les permite tener un contacto directo y afianzar las relaciones familiares.

Sin embargo, que los adultos mayores conozcan el funcionamiento de la internet, así sea porque su familiar migrante se lo haya pedido, es muestra de que dicho saber suple una necesidad comunicativa del migrante con su familia, así no haya una sociabilidad significativa para el grupo familiar, y por consiguiente, para el adulto mayor.

En los hogares donde la internet está presente, el rito consiste en reunir a todo el grupo familiar a conversar y contar qué se ha hecho con el dinero, qué ha pasado en el municipio, qué ha pasado en el hogar y cómo se encuentra el migrante. Le cuentan qué ha cambiado en la casa, qué se puede cambiar, qué no les gusta; como el migrante es el que tiene el poder, se hace lo que él pida. La comunicación es visual (que es una de las ventajas que le reconocen los adultos mayores a la internet).

Al hablarse de ritos se alude a acciones particulares (Finol, 2006), entonces se habla de la familia, que convierte cosas simples en creaciones culturales. Si la internet hiciera parte cotidiana y espontánea de la vida de los adultos mayores y de su familiar migrante, se convertiría en un microuniverso ritual, como es llamado por Finol; pero el proceso adaptativo natural por parte de este grupo no se ha dado, primero, porque algunos hogares montenegrinos con experiencia migratoria carecen de la herramienta; segundo, porque el adulto mayor no tiene la habilidad suficiente para manejarla, y tercero, porque no hay apropiación y no se considera un evento indispensable, sino que hay una predisposición y una planeación para el encuentro, seguidos del hecho de que el poder de la internet como herramienta comunicacional lo tiene una sola persona (nieto, sobrino, amigo cercano).

\section{Conclusiones}

La mayoría de los adultos mayores, es decir personas mayores de sesenta y cinco años del municipio de Montenegro, Quindío, con familiares en el extranjero, han utilizado alguna vez en su vida la internet para comunicarse con sus hijos, nietos, sobrinos u otros familiares que han migrado; este instrumento tecnológico ha sido capaz de facilitar la comunicación entre los migrantes y sus familiares, y les genera sentimientos de alegría y felicidad.

Aunque los adultos mayores prefieren el uso del teléfono fijo y del celular como herramientas para comunicarse debido a la facilidad de su uso, el acceso rápido y los bajos costos en las llamadas, en ocasiones utilizan la internet, ya que esta les permite tener una comunicación sincrónica y completa con sus familiares, les permite verlos, escucharlos y hablarles en el mismo espacio y tiempo.

Esta población no sabe manejar la internet, lo que significa que no tiene ningún tipo de conocimiento y dominio de 
la herramienta y por tal razón, cuando la utilizan nunca lo hacen de manera autónoma e independiente, sino que siempre deben estar acompañados de familiares cercanos o personas capaces de ayudarles (nietos e hijos) y que se convierten en intermediarios de la comunicación.

Los adultos mayores usan la internet pero no se apropian de ella, lo que significa que no hace parte de su vida cotidiana, a pesar de que la consideren un elemento muy importante y significativo en los momentos comunicativos.

Uno de los resultados más importantes de este estudio es que se pudo establecer que las relaciones familiares de los adultos mayores y sus familiares ausentes sí se transforman, pero con el proceso migratorio en sí mismo, es decir desde el momento inicial en que uno de los integrantes de la familia se va para el extranjero dejando su hogar. Esta transformación implica cambios de roles en la composición familiar y en las distribuciones y producciones económicas. La internet y otras tecnologías de la información y la comunicación no transforman las relaciones familiares, pero sí las mantienen, es decir, que por medio de la comunicación se pueden mejorar, empeorar o mantener iguales las relaciones entre las personas que componen una unidad familiar.

\section{Referencias}

Álvarez, S. A. (2000). Jóvenes de corazón: la utilidad de la vejez. Barcelona, España: Ediciones Martínez Roca.

Crovi, D. D. (2007). Dimensión social del acceso, uso y apropiación de las tecnologías de la información y la comunicación. Lima: Contra texto digital, Año 5, ISSN: 19934904.

Dabas, E. \& Najmanovich, D. (1995). Redes, el lenguaje de los vínculos, Ciudadanos de la cibercultura. Buenos Aires: Paidós.
DANE (2005). Censo general 2005. Departamento Administrativo Nacional de Estadística. Bogotá Colombia.

Finol, J. E. (2006). Rito, espacio y poder en la vida cotidiana. Designis.

Jull, J. (1980). Los usos sociales de la comunicación. En Human Comunicación Research 6 (Ed.) y Avendaño, C. (Trad.) . Santiago, Chile: Universidad Diego Portales.

Luhmann, N. (1984). Sistemas sociales, lineamientos para una teoría general. Barcelona: Antrophos.

McLuhan, M. (1996). Comprender los medios de comunicación, las extensiones del ser humano. Barcelona: Paidós Ibérica.

Organización de Naciones Unidas (ONU) Textos extraídos el 15 de Octubre del 2009: http://www.un.org/es/.

Organización Internacional para las Migraciones. (2006). Glosario sobre migración, derecho internacional sobre migración. (7). Recuperado de http://publications.iom. int/system/files/pdf/iml_7_sp.pdf.

Páez Morales, Guillermo, (1984), Sociología de la Familia, Elementos de Análisis en Colombia y América Latina, Universidad Santo Tomás, Centro de Enseñanza Descentralizada.

RuizElisa Dulcey, Cecilia UribeValdivieso, (2002) Psicología del Ciclo Vital: Hacia una Visión Comprehensiva de la Vida Humana, Revista Latinoamericana de Psicología, Universidad Javeriana, Bogotá Colombia. Pág. 19.

Sánchez $g$ (s.f.) El Adulto Mayor en la familia, Extraído el 28 de Septiembre de 2009, http://www. esperanzaparalafamilia.com. 